\title{
A Model for Types and Levels of Human Interaction with Automation
}

\author{
Raja Parasuraman, Thomas B. Sheridan, Fellow, IEEE, and Christopher D. Wickens
}

\begin{abstract}
Technical developments in computer hardware and software now make it possible to introduce automation into virtually all aspects of human-machine systems. Given these technical capabilities, which system functions should be automated and to what extent? We outline a model for types and levels of automation that provides a framework and an objective basis for making such choices. Appropriate selection is important because automation does not merely supplant but changes human activity and can impose new coordination demands on the human operator. We propose that automation can be applied to four broad classes of functions: 1) information acquisition; 2) information analysis; 3) decision and action selection; and 4) action implementation. Within each of these types, automation can be applied across a continuum of levels from low to high, i.e., from fully manual to fully automatic. A particular system can involve automation of all four types at different levels. The human performance consequences of particular types and levels of automation constitute primary evaluative criteria for automation design using our model. Secondary evaluative criteria include automation reliability and the costs of decision/action consequences, among others. Examples of recommended types and levels of automation are provided to illustrate the application of the model to automation design.
\end{abstract}

Index Terms-Automation, cognitive engineering, function allocation, human-computer interaction, human factors, human-machine systems, interface design.

\section{INTRODUCTION}

C ONSIDER the following design problem. A human operator of a complex system provided with a large number of dynamic information sources must reach a decision relevant to achieving a system goal efficiently and safely. Examples include an anesthesiologist given various vital signs who must decide whether to increase the dosage of a drug to a patient undergoing surgery; an air defense operator given various sensor readings who has to decide whether to shoot down a potentially hostile enemy aircraft; or a securities analyst given various financial data who must judge whether to buy a large block of stocks. Technical developments in computer hardware and software make it possible to automate many aspects of the system, i.e., to have a computer carry out certain functions that the human operator would normally perform. The automation

Manuscript received January 26, 1999; revised February 7, 2000. This work was supported by grants from NASA Ames Research Center, Moffett Field, CA (NAG-2-1096) and NASA Goddard Space Research Center, MD (NAG 5-8761) to R.P., and from NASA Ames Research Center to T.B.S. (NAG-2-729). This paper was recommended by Associate Editor R. Rada.

R. Parasuraman is with the Cognitive Science Laboratory, The Catholic University of America, Washington, DC 20064 USA.

T. B. Sheridan is with the Massachusetts Institute of Technology, Cambridge, MA 02165 USA.

C. D. Wickens is with the University of Illinois, Champaign, IL 61820 USA. Publisher Item Identifier S 1083-4427(00)03579-7. can differ in type and complexity, from simply organizing the information sources, to integrating them in some summary fashion, to suggesting decision options that best match the incoming information, or even to carry out the necessary action.

The system design issue is this: given these technical capabilities, which system functions should be automated and to what extent? These fundamental questions increasingly drive the design of many new systems. In this paper we outline a model of human interaction with automation that provides a framework for answers to these questions. The human performance consequences of specific types and levels of automation constitute the primary evaluative criteria for automation design using the model. Secondary evaluative criteria include automation reliability and the costs of action consequences. (Both these sets of criteria are described more fully later in this paper). Such a combined approach — distinguishing types and levels of automation and applying evaluative criteria-can allow the designer to determine what should be automated in a particular system. Because the impact of the evaluative criteria may differ between systems, the appropriate types and levels of automation for different systems can vary widely. Our model does not therefore prescribe what should and should not be automated in a particular system. Nevertheless, application of the model provides a more complete and objective basis for automation design than do approaches based purely on technological capability or economic considerations.

\section{Automation}

Machines, especially computers, are now capable of carrying out many functions that at one time could only be performed by humans. Machine execution of such functions-or automation-has also been extended to functions that humans do not wish to perform, or cannot perform as accurately or reliably as machines. Technical issues-how particular functions are automated, and the characteristics of the associated sensors, controls, and software-are major concerns in the development of automated systems. This is perhaps not surprising given the sophistication and ingenuity of design of many such systems (e.g., the automatic landing of a jumbo jet, or the docking of two spacecraft). The economic benefits that automation can provide, or are perceived to offer, also tend to focus public attention on the technical capabilities of automation.

In contrast to the voluminous technical literature on automation, there is a small but growing research base examining the human capabilities involved in work with automated systems [1]-[8]. This work has shown clearly that automation does not simply supplant human activity but rather changes it, often in 
ways unintended and unanticipated by the designers of automation [8], and as a result poses new coordination demands on the human operator [7]. Until recently, however, these findings have not had much visibility or impact in engineering and design circles. Examination of human performance issues is especially important because modern technical capabilities now force system designers to consider some hard choices regarding what to automate and to what extent, given that there is little that cannot be automated. In the present paper we propose a model for types and levels of automation that provides a framework and an objective basis for making such choices. Our approach was guided by the concept of "human-centered automation" [9] and by a previous analysis of automation in air traffic control (ATC) [10]. ${ }^{1}$

Let us begin by defining automation, because the term has been used many different ways. The Oxford English Dictionary (1989) defines automation as

1) Automatic control of the manufacture of a product through a number of successive stages;

2) the application of automatic control to any branch of industry or science;

3) by extension, the use of electronic or mechanical devices to replace human labor.

The original use of the term implies automatic control (automatic having many alternative definitions suggesting reflexive action, spontaneity, and independence of outside sources). Automatic control can be open loop as well as closed loop, and can refer to electronic as well as mechanical action. Automation does not simply refer to modernization or technological innovation. For example, updating a computer with a more powerful system does not necessarily constitute automation, nor does the replacement of electrical cables with fiber optics. The present paper is concerned with human performance in automated systems. We therefore use a definition that emphasizes human-machine comparison and define automation as a device or system that accomplishes (partially or fully) a function that was previously, or conceivably could be, carried out (partially or fully) by a human operator [8].

\section{A MODEL FOR TYPES AND LEVELS OF AUTOMATION}

In our definition, automation refers to the full or partial replacement of a function previously carried out by the human operator. This implies that automation is not all or none, but can vary across a continuum of levels, from the lowest level of fully manual performance to the highest level of full automation. Several levels between these two extremes have been proposed [11], [12]. Table I shows a 10-point scale, with higher levels representing increased autonomy of computer over human action [10], based on a previously proposed scale [11]. For example, at a low level 2, several options are provided to the human, but the system has no further say in which decision is chosen. At level 4 , the computer suggests one decision alternative, but the human

\footnotetext{
${ }^{1}$ In principle, our approach does not exclude the possibility of full automation, without any human operator involvement. This might suggest that our model is not needed if total automation is technically feasible. As we discuss later, however, full automation does not necessarily eliminate a human role in automated systems [8].
}

TABLE I

LEVELS OF AUTOMATION OF DECISION AND ACTION SELECTION
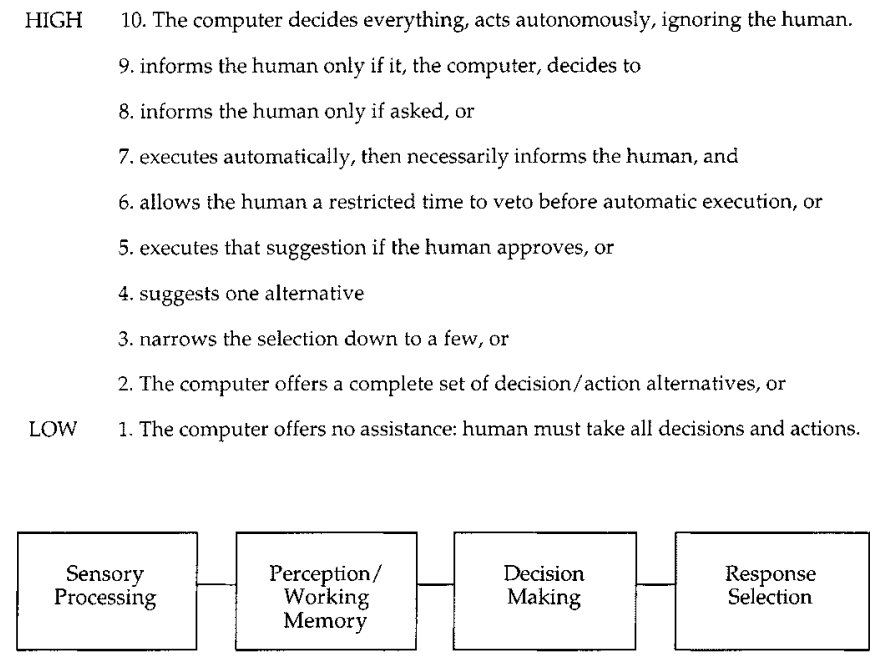

Fig. 1. Simple four-stage model of human information processing.

retains authority for executing that alternative or choosing another one. At a higher level 6, the system gives the human only a limited time for a veto before carrying out the decision choice.

Automated systems can operate at specific levels within this continuum. For example, a conflict detection and resolution system that notifies an air traffic controller of a conflict in the flight paths of two aircraft and suggests a resolution would qualify as level 4 automation. Under level 6 or higher, the system would automatically execute its own resolution advisory, unless the controller intervened.

In the proposed model we extend Table I to cover automation of different types of functions in a human-machine system. The scale in Table I refers mainly to automation of decision and action selection, or output functions of a system. However, automation may also be applied to input functions, i.e., to functions that precede decision making and action. In the expansion of the model, we adopt a simple four-stage view of human information processing (see Fig. 1).

The first stage refers to the acquisition and registration of multiple sources of information. This stage includes the positioning and orienting of sensory receptors, sensory processing, initial pre-processing of data prior to full perception, and selective attention. The second stage involves conscious perception, and manipulation of processed and retrieved information in working memory [13]. This stage also includes cognitive operations such as rehearsal, integration and inference, but these operations occur prior to the point of decision. The third stage is where decisions are reached based on such cognitive processing. The fourth and final stage involves the implementation of a response or action consistent with the decision choice.

This four-stage model is almost certainly a gross simplification of the many components of human information processing as discovered by information processing and cognitive psychologists [14]. The performance of most tasks involves inter-dependent stages that overlap temporally in their processing operations [15]. The stages can also be considered to be coordinated 


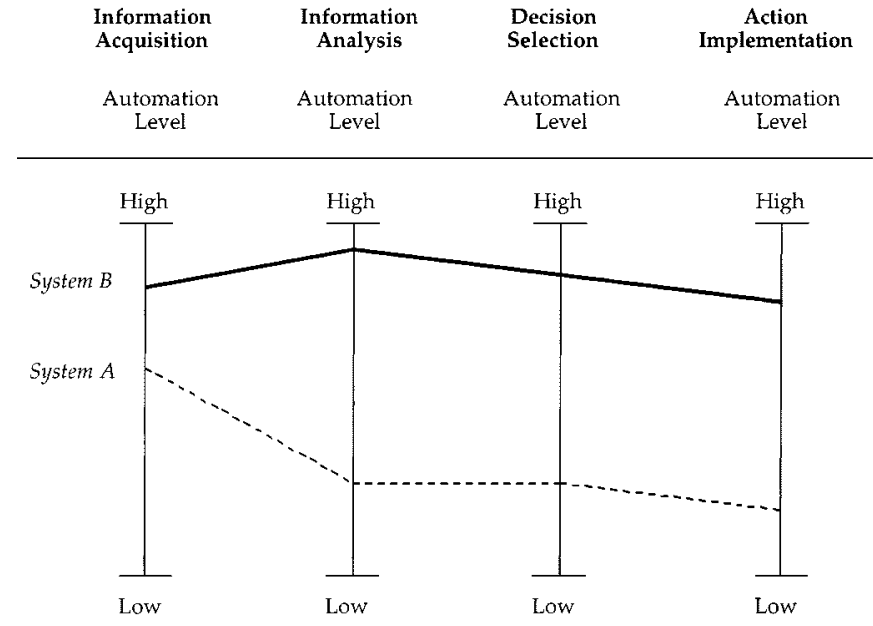

Fig. 2. Levels of automation for independent functions of information acquisition, information analysis, decision selection, and action implementation. Examples of systems with different levels of automation across functional dimensions are also shown.

together in "perception-action" cycles [16] rather than in a strict serial sequence from stimulus to response. Our goal is not to debate the theoretical structure of the human cognitive system but to propose a structure that is useful in practice. In this respect, the conceptualization shown in Fig. 1 provides a simple starting point with surprisingly far-reaching implications for automation design. Similar conceptual models have been found to be useful in deriving human factors recommendations for designing systems in general [17].

The four-stage model of human information processing has its equivalent in system functions that can be automated. Accordingly, we propose that automation can be applied to four classes of functions (see also [18] and related proposals in [9] and [19]):

1) information acquisition;

2) information analysis;

3) decision and action selection;

4) action implementation.

Each of these functions can be automated to differing degrees, or many levels. The multiple levels of automation of decision making as shown in Table I can be applied, with some modification, to the information acquisition, information analysis, and action implementation stages as well, although the number of levels will differ between the stages. Fig. 2 provides a schematic of our model of types and levels of automation. As a convenient shorthand, we refer to the four types as acquisition, analysis, decision, and action automation. We also occasionally refer jointly to acquisition and analysis automation as information automation.

A particular system can involve automation of all four dimensions at different levels. Thus, for example, a given system (A) could be designed to have moderate to high acquisition automation, low analysis automation, low decision automation, and low action automation. Another system (B), on the other hand, might have high levels of automation across all four dimensions.

\section{A. Acquisition Automation}

Automation of information acquisition applies to the sensing and registration of input data. These operations are equivalent to the first human information processing stage, supporting human sensory processes. At the lowest level, such automation may consist of strategies for mechanically moving sensors in order to scan and observe. For example, the radars used in commercial ATC acquire information on aircraft by scanning the sky in a fixed pattern, but in military ATC the radars may "lock on" as a function of detected targets. Artificial visual and haptic sensors could also be used with an industrial robot to allow it to find and grasp an object, thereby providing information about that object. Moderate levels of automation at this stage may involve organization of incoming information according to some criteria, e.g., a priority list, and highlighting of some part of the information. For example "electronic flight strips" for air traffic controllers could list aircraft in terms of priority for handling; and the electronic data block showing aircraft on the controller's radar display (which itself represents an earlier form of acquisition automation) could be highlighted to indicate a potential problem with a particular aircraft. Note that both organization and highlighting preserve the visibility of the original information ("raw" data). This is not necessarily the case with a more complex operation at this stage of automation, filtering, in which certain items of information are exclusively selected and brought to the operator's attention. Highlighting and filtering can lead to differing human performance consequences, as described in a later section in a discussion of automation reliability.

\section{B. Analysis Automation}

Automation of information analysis involves cognitive functions such as working memory and inferential processes. At a low level, algorithms can be applied to incoming data to allow for their extrapolation over time, or prediction. For example, predictor displays have been developed for the cockpit that show the projected future course of another aircraft in the neighboring airspace [20], [21]. Trend displays have also been developed for use in process control (e.g., nuclear power plants), in which a model of the process is developed and used to show both the current and the anticipated future state of the plant [22]. A higher level of automation at this stage involves integration, in which several input variables are combined into a single value. One example is to use a display with an emergent perceptual feature such as a polygon against a background of lines [23]. Another example of information analysis automation in ATC is the converging runway display aid (CRDA), which eliminates the need for the controller to mentally project the approach path of one aircraft onto that of another landing on a converging runway [24]. In both these examples, information integration serves the purpose of augmenting human operator perception and cognition. More complex forms of analysis automation include "information managers" that provide context-dependent summaries of data to the user [45].

\section{Decision Automation}

The third stage, decision and action selection, involves selection from among decision alternatives. Automation of this 
stage involves varying levels of augmentation or replacement of human selection of decision options with machine decision making, as described previously in Table I. For example expert systems are designed with conditional logic (i.e., production rules) to prescribe a specific decision choice if particular conditions exist [25]. Examples can be found in medicine [26], military command and control [27], and in route planning for pilots to avoid bad weather [28]. As with the analogous decision-making stage in human performance, such systems depart from those involved in inference (analysis automation) because they must make explicit or implicit assumptions about the costs and values of different possible outcomes of the decision process, and the nature of these outcomes is uncertain in a probabilistic world. The different levels of automation at this stage are best defined by the original taxonomy proposed by Sheridan [11] and shown in Table I, which defines a continuum that progresses from systems that recommend courses of action, to those that execute those courses. For example, in comparing proposed and existing designs for decision automation in avoiding aircraft-ground collisions, the current ground proximity warning system (GPWS) is positioned at level 4 , in which a single maneuver is recommended, but the pilot can chose to ignore it. But a proposed automatic ground collision avoidance (auto GCAS) system for combat aircraft is defined at level 7, in which automation will automatically take control if the pilot does not [29].

\section{Action Automation}

The final stage of action implementation refers to the actual execution of the action choice. Automation of this stage involves different levels of machine execution of the choice of action, and typically replaces the hand or voice of the human. Different levels of action automation may be defined by the relative amount of manual versus automatic activity in executing the response. For example, in a photocopier, manual sorting, automatic sorting, automatic collation, and automatic stapling represent different levels of action automation that can be chosen by the user. A somewhat more complex example from ATC is the automated "handoff," in which transfer of control of an aircraft from one airspace sector to another is carried out automatically via a single key press, once the decision has been made by the controller. On the flight deck, systems are also being considered in which a flight plan, uplinked from the ground, can be "autoloaded" into the plane's flight management computer by a single keypress, rather than through more time-consuming manual data entry [30]-[32]. Finally, action automation includes "agents" that track user interaction with a computer and execute certain sub-tasks automatically in a contextually-appropriate manner [45].

\section{E. Adaptive Automation}

Levels of automation across any of these functional types need not be fixed at the system design stage. Instead, the level (and perhaps even the type) of automation could be designed to vary depending on situational demands during operational use. Context-dependent automation is known as adaptive automation [33]-[35]. Two examples will illustrate the concept. In an air defense system, the beginning of a "pop-up" weapon delivery sequence could lead to the automation at a high level of all aircraft defensive measures [36]. The automation is adaptive because if this critical event does not occur, the automation is not invoked or is set at a low level. In another example, the decision to continue or abort an aircraft takeoff following an engine malfunction might be automated at either a low or a high level depending upon the time criticality of the situation (e.g., how close the aircraft is to the critical speed V1 for takeoff) [37]. Considerable empirical research on adaptive automation has been reported in recent years [38]-[44]. However, we do not describe this work because it raises several complex ancillary issues, the discussion of which would take us far afield from the primary purpose of this paper.

\section{A Framework for Automation Design}

The model we have outlined provides a framework for examining automation design issues for specific systems. How can the framework be used? We propose a series of steps and an iterative procedure that can be captured in a flow chart (see Fig. 3). The first step is to realize that automation is not all-or-none but can vary by type. One can ask whether automation should be applied to information acquisition, information analysis, decision selection, or to action implementation. Automation of one class of function (e.g., information analysis), of different combinations of functions, or of all four functional domains, can be entertained.

At a subsequent stage of design, one can ask what level of automation should be applied within each functional domain. There is probably no simple answer to this question, and tradeoffs between anticipated benefits and costs are likely. However, the four-dimensional model we have proposed can provide a guiding framework. As shown in Fig. 3, multiple levels of automation can be considered for each type of automation. We propose that any particular level of automation should be evaluated by examining its associated human performance consequences. These constitute primary evaluative criteria for levels of automation. However, human performance is not the only important factor. Secondary evaluative criteria include automation reliability and the costs of decision/action consequences ${ }^{2}$. These should also be applied to evaluate the feasibility and appropriateness of particular levels of automation. We envisage the application of these criteria and their evaluation as constituting a recursive process (see Fig. 3) that could be made part of an iterative design procedure. We emphasize, however, that the model should not be treated as a static formula or as a prescription that decrees a particular type or level of automation. Rather, when considered in combination with the primary and secondary evaluative criteria we have described, the model can provide principled guidelines for automation design.

We provide examples where, following consideration of these evaluative criteria, particular levels of automation are recommended for each of the four types or stages of automation. Such recommendations refer to the appropriate upper bound on the level of automation, i.e., the maximum, but not necessarily the required level. In other words, we recommend that automation

\footnotetext{
${ }^{2}$ This is not an exhaustive list of criteria. Others that are important include ease of system integration, efficiency/safety tradeoffs, manufacturing and operating costs, and liability issues.
} 


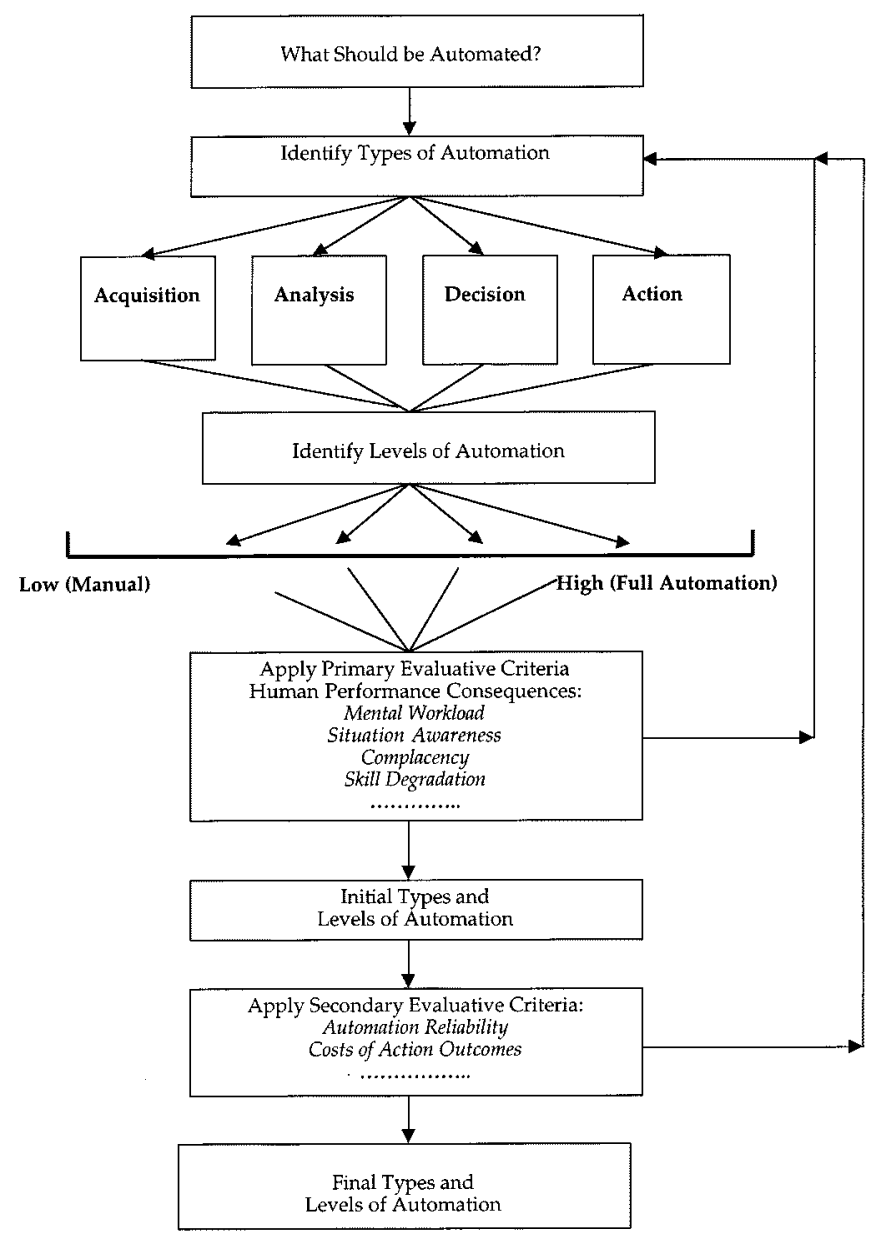

Fig. 3. Flow chart showing application of the model of types and levels of automation. For each type of automation (acquisition, analysis, decision, and action), a level of automation between low (manual) and high (full automation) is chosen. This level is then evaluated by applying the primary evaluative criteria of human performance consequence, and adjusted if necessary, in an iterative manner as shown. Secondary evaluative criteria are then also iteratively applied to adjust the level of automation. The process is then repeated for all four types of automation.

could be designed to go as high as that particular level, but no further. But the designer could choose a level lower than this maximum if necessary, particularly after considering evaluative criteria other than the ones we discuss (e.g., ease of system integration, or cost). The lower bound on the level of automation can also be determined by applying the same evaluative criteria. Acceptable system performance may require a certain minimal level of automation.

\section{A. Human Performance Consequences: Primary Evaluative Criteria for Automation Design}

An important consideration in deciding upon the type and level of automation in any system design is the evaluation of the consequences for human operator performance in the resulting system (i.e., after automation has been implemented). As shown in Fig. 3, particular types and levels of automation are evaluated by examining their associated human performance consequences. To take a hypothetical example, suppose prior research has shown (or modeling predicts) that compared to manual operation, both human and system performance are enhanced by level 4 automation but degraded by automation above level 6 . Application of our framework would determine the lower and upper bounds of automation to be 4 and 6 , respectively. This initial specification would then be evaluated again with respect to the secondary evaluative criteria, in an iterative manner, and a final choice of level within this range could be made (see Fig. 3).

Over the past two decades, researchers have examined a number of different aspects of human interaction with automated systems. This research, which has included theoretical analyzes, laboratory experiments, simulation and modeling, field studies, and analyzes of real-world incidents and accidents, has found that automation can have both beneficial and negative effects on human performance [1]-[10], [45]-[48]. We briefly discuss four human performance areas: mental workload, situation awareness, complacency, and skill degradation.

1) Mental Workload: The evidence suggests that well-designed information automation can change human operator mental workload to a level that is appropriate for the system tasks to be performed. At the simplest level, organizing information sources, e.g., in a priority list, will help the operator in picking the information relevant to a decision. Data summaries can also help by eliminating time-consuming search or communication operations. As mentioned previously, the electronic data block on the air traffic controller's radar display replaces the need for the controller to communicate with pilots to determine the aircraft position and altitude. Other information automation operations that are beneficial include highlighting, and integration, in which different information sources are collated and presented together [10]. Cockpit predictor displays have also shown that pilot workload decreases and hazard detection performance improves with the addition of predictive information concerning the flight path of neighboring aircraft [21]. Data transformation, for example graphic presentation of information, can also be beneficial. Transformation and integration of raw data into a form (graphical or otherwise) that matches the operator's representation of system operations has been found to be a useful design principle [49]. A good example is the horizontal situation indicator in the cockpit, which provides the pilot with a graphic display of the projected flight plan and the current position of the aircraft. This, more than any other automated system in the cockpit, has been credited with reducing the workload of the pilot [50].

These results should not be construed to mean that automation always results in balanced operator workload. Instances of automation increasing workload have also been found [8], [50]. These mostly involve systems in which the automation is difficult to initiate and engage, thus increasing both cognitive workload [51] and if extensive data entry is required, the physical workload of the operator. Such systems have been referred to as implementing "clumsy" automation [50]. In general, the effect of automation on mental workload has been mirrored by the similarly mixed record of automation in improving human productivity and efficiency [52].

In addition to unbalanced mental workload, other human performance costs have been linked to particular forms of automation. We briefly consider three such costs.

2) Situation Awareness: First, automation of decision-making functions may reduce the operator's awareness 
of the system and of certain dynamic features of the work environment. Humans tend to be less aware of changes in environmental or system states when those changes are under the control of another agent (whether that agent is automation or another human) than when they make the changes themselves [53]-[56]. Also, if a decision aid, expert system, or other type of decision automation consistently and repeatedly selects and executes decision choices in a dynamic environment, the human operator may not be able to sustain a good "picture" of the information sources in the environment because he or she is not actively engaged in evaluating the information sources leading to a decision. This might occur in systems where operators act as passive decision-makers monitoring a process to determine when to intervene so as to prevent errors or incidents [53]. Note that such a cost may occur even as the use of automation of information analysis, e.g., data integration, may improve the operator's situation awareness.

3) Complacency: Second, if automation is highly but not perfectly reliable in executing decision choices, then the operator may not monitor the automation and its information sources and hence fail to detect the occasional times when the automation fails [57], [58]. This effect of over-trust or "complacency" is greatest when the operator is engaged in multiple tasks and less apparent when monitoring the automated system is the only task that the operator has to perform [58]. The complacency effect in monitoring has recently been modeled using a connectionist architecture [59]: the analysis suggested that complacency reflects differential learning mechanisms for monitoring under manual control and automation.

Automation of information analysis can also lead to complacency if the algorithms underlying filtering, prediction, or integration operations are reliable but not perfectly so. A recent study of a simulated air-ground targeting task [60] found that a cue that incorrectly directed attention away from the target led to poorer detection performance even though pilots were informed that the cue was not perfectly reliable. Automated cueing (attention guidance) can lead operators to pay less attention to uncued areas of a display than is appropriate [61]. Thus complacency-like effects can also be obtained even if automation is applied to information acquisition and analysis and not just to decision-making. It is not known, however, whether such effects of unreliable automation apply equally strongly to all stages of information processing. There is some evidence to indicate that although complacency can occur with both information automation and decision automation, its effects on performance are greater with the latter. In a study of decision aiding, both forms of automation benefited performance equally when the automation was perfectly reliable [62]. When the automation was unreliable, however, performance suffered much more when unreliable recommendations were given by decision automation than when only incorrect status information was provided by information automation. This study, however, is the only one to date that has directly compared the effects of automation unreliability at different stages of automation. The issue of whether automation unreliability has similar negative effects for all four stages of automation in our model needs further examination.

4) Skill degradation: Third, if the decision-making function is consistently performed by automation, there will come a time when the human operator will not be as skilled in performing that function. There is a large body of research in cognitive psychology documenting that forgetting and skill decay occur with disuse [63]. Degradation of cognitive skills may be particularly important following automation failure. A recent simulation study of human control of a telerobotic arm used for movement of hazardous materials found that following automation malfunction, performance was superior with an intermediate level of decision automation compared to higher levels [53].

These potential costs-reduced situation awareness, complacency, and skill degradation-collectively demonstrate that high-level automation can lead to operators exhibiting "out-of-the-loop" unfamiliarity [47]. All three sources of vulnerability may pose a threat to safety in the event of system failure. Automation must therefore be designed to ensure that such potential human performance costs do not occur. Human performance costs other than the areas we have discussed should also be examined. Automation that does not lead to unbalanced mental workload, reduced situation awareness, complacency, or skill loss may nevertheless be associated with other human performance problems that ultimately impact on system performance, including mode confusion and low operator trust in automation [1]-[10], [45]-[48].

By considering these human performance consequences, the relative merits of a specific level of automation can be determined. However, full application of our model also requires consideration of other criteria. We consider two other secondary criteria here, automation reliability and the cost of decision and action outcomes.

\section{B. Secondary Evaluative Criteria}

1) Automation Reliability: The benefits of automation on operator mental workload and situation awareness noted previously are unlikely to hold if the automation is unreliable. Hence ensuring high reliability is a critical evaluative criterion in applying automation. Several procedures for estimating reliability have been proposed, including fault and event tree analysis [64] and various methods for software reliability analysis [65]. The use of these techniques can be helpful, so long as their results are interpreted cautiously. In particular, what appear to be "hard numbers," such as a reliability of .997 , or a mean time to failure of 100000 hours, must be viewed with some skepticism because such values represents an estimate of a mean, whereas what is required is the variance around the mean, which can be considerable. The complexity and size of software in many automated systems may also preclude comprehensive testing for all possible faults, particularly those that arise from interaction with the existing system in which the automated sub-system is placed [10]. Furthermore, automation reliability cannot always simply be defined in probabilistic terms. Failures may occur not because of a predictable (in a statistical sense) malfunction in software or hardware, but because the assumptions that are modeled in the automation by the designer are not met in a given operational situation [8].

Automation reliability is an important determinant of human use of automated systems because of its influence on human trust [66] [67]. Unreliability lowers operator trust and can therefore undermine potential system performance benefits of the 
automation. Automated systems may be underutilized or disabled because of mistrust, as in the case of alarm systems that frequently give false alerts [8]. Signal detection analysis [68] can be used to determine the alerting threshold that balances the competing requirements of timely detection (to allow for effective action), a near-zero missed detection rate (because of potentially catastrophic consequences-e.g., a collision), and a low false alert rate [69]. To ensure alert reliability, the probability that an alarm reflects a true hazardous event must also be maximized to the extent possible: this can be examined by combining signal detection theory and Bayesian statistics [70].

If information automation can be made extremely reliable, then pursuing very high levels of information automation can be justified. Of course, high reliability cannot be guaranteed in many cases. As mentioned previously, the inherent uncertain nature of information sources, either due to sensor imprecision or to changes in operator priorities, means that there will always exist conditions in which the algorithms used by the automation are inappropriate for those conditions. Nevertheless, information acquisition and analysis automation may still be retained at a relatively high level, as long as the operator has access to the raw data (e.g., highlighting, but not filtering), and the operator is aware of (calibrated to) the level of unreliability, such that some attention will be allocated to the original information [60], [71].

Although many examples of highly reliable information automation exist, more sophisticated forms of such automation are being developed in which complex algorithms are applied to the raw data in order to predict future events. For example, traffic displays in the cockpit, and conflict prediction tools for the air traffic controller both attempt to project the future flight paths of aircraft. Projecting the future is inherently less than perfectly reliable, particularly if carried out far enough out in time (e.g., $20 \mathrm{~min}$. for ATC conflict prediction). Further work needs to be done to evaluate not only the reliability of the algorithms underlying these predictor systems, but also their susceptibility to noise in the raw data, and the consequences for human performance of information automation unreliability. Some emerging research is beginning to define the conditions under which unreliability does or does not influence human performance. For example, two recent studies found that when feedback is provided as to the occasional errors made by information automation, appropriate calibration of the operator's trust in the automation can take place fairly rapidly, and the benefits of information automation can still be realized [60], [71]. This suggests that the negative effects of over-trust, noted earlier for decision automation, may be less apparent for information automation. However, as discussed previously, only one study has directly compared information and decision automation [62]. Thus the issue of whether automation unreliability has greater negative effects for later stages of automation requires further examination.

2) Costs of Decision/Action Outcomes: Our analysis so far indicates that high levels of automation may be associated with potential costs of reduced situation awareness, complacency, and skill degradation. This is not to say that high levels of automation should not be considered for decision and action automation. However, assessing the appropriate level of automation for decision automation requires additional consideration of the costs associated with decision and action outcomes.
The decisions and associated actions that humans and automated systems take in most systems vary in the costs that occur if the actions are incorrect or inappropriate. Many routine actions have predictable consequences that involve little or no cost if the actions do not go as planned. The risk associated with a decision outcome can be defined as the cost of a error multiplied by the probability of that error. For decisions involving relatively little risk, therefore, out-of-the-loop problems are unlikely to have much impact, even if there is a complete automation failure. Such decisions are strong candidates for high-level automation. In fact, if human operators had to be continually involved in making each of these relatively simple decisions, they could be so overloaded as to prevent them from carrying out other more important functions.

Note that high-level automation of decision selection and action may also be justified in highly time-critical situations in which there is insufficient time for a human operator to respond and take appropriate action. For example, if certain serious problems are detected in the reactor of a nuclear power plant, control rods are automatically lowered into the core to turn off the reactor, without any human operator intervention. Bypassing the human operator is justified in this case because the operator cannot reliably respond in time to avoid an accident. As previously discussed, automating the decision to abort or continue the takeoff of an aircraft when an engine malfunction occurs too near in time to the critical V1 speed for appropriate pilot action would represent another qualifying example [37], as would the decision to take control of the aircraft if a fighter aircraft is about to run into the ground [29].

It is also appropriate to consider high-level automation for decisions involving high risk in situations in which human operators have time to respond. In this case, the cost of adverse consequences define major evaluative criteria for determining appropriate levels of automation. The examples in anesthesiology, air defense, and the stock market with which we began this paper qualify as involving high-cost decisions. System designers can certainly consider implementing decision automation above low to moderate levels for such systems, e.g., at levels at or above level 6 in Table I, in which computer systems are given autonomy over decision making. This would be appropriate if the human operator is not required to intervene or manage the system in the event of automation failure. In fact, in this case even full automation (Level 10) could be justified ${ }^{3}$. However, if the human operator is ever expected under abnormal circumstances to take over control, then our analysis suggests that high levels of decision automation may not be suitable because of the documented human performance costs associated with such automation. The burden of proof should then be on the designer to show that their design will not lead to the problems of loss of situation awareness, complacency, and skill loss that we have discussed.

${ }^{3}$ Full automation requires highly reliable error handling capabilities and the ability to deal effectively and quickly with a potentially large number of anomalous situations. In addition to requiring the technical capability to deal with all types of known errors, full automation without human monitoring also assumes the ability to handle unforeseen faults and events. This requirement currently strains the ability of most intelligent fault-management systems. 
A system designer may object to the recommendation that decision automation should not exceed a moderate level for high-risk situations on the grounds that if information automation can be made highly reliable, then decision automation can also be, so why not implement high-level automation for this function too? The answer is that although decision-aiding systems can be engineered to be highly reliable for many known conditions, the "noisiness" of the real world, with unplanned variations in operating conditions, unexpected or erratic behavior of other system components or human operators, system malfunctions, etc., as well as the inherent unreliability of predicting the future, will mean that there will always be a set of conditions under which the automation will reach an incorrect decision. If under such conditions of system failure the human operator is required to intervene and salvage the situation, the problem of out-the-loop unfamiliarity may prevent the operator from intervening successfully or in a timely manner [8], [47], [55].

Finally, the inter-dependence of the decision automation and action automation dimensions for high-risk functions should be noted. A system could be designed to have high-level decision automation, in which decision choices are selected without human involvement or veto power. For example, currently an air traffic controller issues a verbal clearance to a pilot, who acknowledges and then executes a flight maneuver consistent with the clearance. With the development of two-way electronic data link communications between aircraft and ATC, however, the clearance (which itself may be a computer choice) could be uplinked and loaded in the aircraft's flight management system (FMS) automatically. The aircraft could then carry out the maneuver, without pilot intervention. If the consequences of an incorrect or inappropriate decision are great, however, then it would be prudent to require that the action automation level be sufficiently low so that the (automated) decision choice is executed by the pilot (i.e., by actively pressing a button that "loads" the proposed flight plan into the FMS). Giving the pilot the opportunity to review the decision choice and forcing a conscious overt action, provides an "error-trapping" mechanism that can guard against mindless acquiescence in computer-generated solutions that are not contextually appropriate. Note that we are not implying that some degree of human action is always needed for the purposes of error trapping. The need only arises at the last action implementation stage if the previous decision selection stage has been highly automated. In this situation having some human involvement at the action stage provides a "last chance opportunity" to trap errors.

Recent studies have examined the relative effects of low and high levels of action automation on use of the FMS [30], [31]. Use of a lower level of automation of action selection-in entering data-linked flight information into the flight management computer-allowed for more errors of decision making automation to be caught, than a higher level, in which data entry was accomplished by pressing a single "accept" button. Of course this advantage for error trapping must be balanced against the added workload, and possible error source of less automated (manual) data entry [32]. Certainly cumbersome and clumsy data entry remains a viable candidate for automation. But to reiterate the linkage between decision and action automation, if high au- tomation is selected for the latter, then designers should resist the temptation for high automation levels of decision making.

\section{Application Example}

Our multi-stage model of human-automation interaction can be applied to specific systems in conjunction with a consideration of evaluative criteria, of which we have discussed three in this paper-human performance consequences, automation reliability, and the costs of decision/action consequences. To further illustrate application of the model, we briefly consider its use in the design of future ATC systems, based on analyses previously presented in [10].

ATC systems are being redesigned because the volume of air traffic is likely to double over the next two decades, posing a significant threat to handling capacity [72]. One alternative is Free Flight [73], which would allow user-preferred routing and free maneuvering, among other changes aimed at minimizing ATC restrictions [74]. Another approach is to supplement the current system of ground-based ATC with additional automation to support air traffic controllers in the management of an increasingly dense airspace [10]. Elements of both alternatives are likely to be implemented, but the increasing complexity of future airspace will require automation tools to support both air traffic controllers and pilots. Automation tools will be needed for planning, traffic management, conflict detection and resolution, etc.

Application of our model suggests the following recommendations for future ATC automation. (We again emphasize that each recommendation represents an upper bound or maximum level of automation, not a required level.) High levels of information acquisition and analysis automation can be pursued and implemented if the resulting system can be shown to be reliable. This recommendation is represented by the arrows on the left part of the scales in Fig. 4. Several examples of such automation (such as CRDA) already exist and others are being developed. For decision and action automation, however, high levels should be implemented only for low-risk situations (indicated by the upper arrow in the middle scale in Fig. 4). For all other situations, the level of decision automation should not exceed the level of the computer suggesting (but not executing) a preferred alternative to the controller (indicated by the lower arrow). For example, in risky situations, as when a climb clearance has to be issued to resolve a crossing conflict in dense airspace, conflict resolution automation can provide alternatives to the controller but should not select one of them without controller involvement. If relatively high-level decision automation is implemented in risky situations, however, then we recommend that some degree of human action be retained by having a moderate level of action automation. As discussed previously, this allows for last-stage error trapping. This recommendation is indicated by the right-most arrow in Fig. 4.

\section{Alternatives, Limitations, AND Extensions}

Before concluding, we briefly consider two alternative approaches to the implementation of automation, and discuss some limitations and extensions of our framework. One alternative to our approach is to automate everything that one can. This can be 


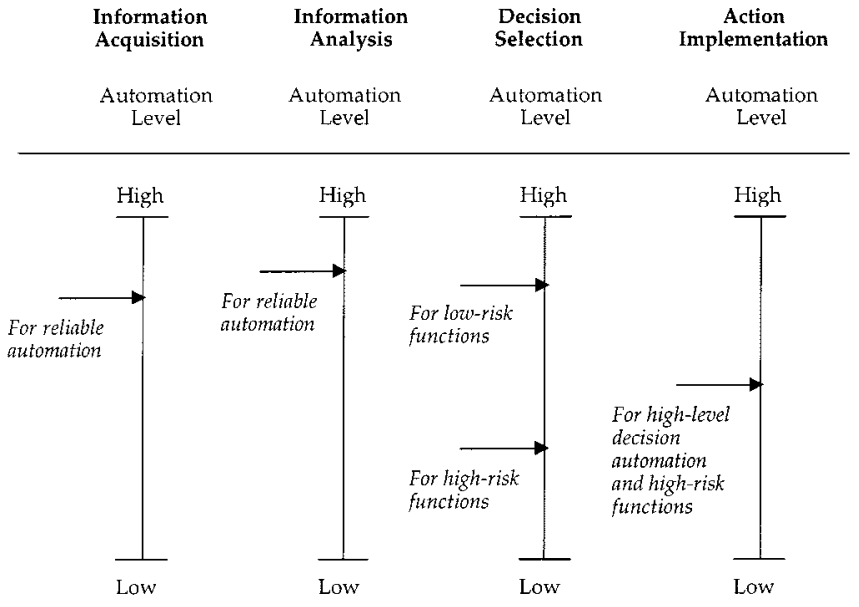

Fig. 4. Recommended types and levels for future ATC systems, consistent with three evaluative criteria-human performance consequences, automation reliability, and costs of actions.

a viable option and to some extent has been the default strategy used in most systems that have been automated to date, often because increasing efficiency or reducing costs are major driving forces for automation. However, a problem with this strategy is that the human operator is left with functions that the designer finds hard, expensive, or impossible to automate (until a cleverer designer comes around). This approach therefore defines the human operator's roles and responsibilities in terms of the automation [8]. Designers automate every subsystem that leads to an economic benefit for that subsystem and leave the operator to manage the rest. Technical capability or low cost are valid reasons for automation, given that there is no detrimental impact on human performance in the resulting whole system, but this is not always the case. The sum of subsystem optimizations does not typically lead to whole system optimization. A second alternative is to use task allocation methods to match human and machine capabilities, as in the Fitts list approach [75]. That is, tasks that are putatively performed better by machines should be automated, whereas those that humans do better should not. Unfortunately, although function allocation methods are useful in principle, it has proved difficult in practice to use procedures such as the Fitts List to determine which functions should be automated in a system [76].

Some limitations of our model for types and levels of automation should also be noted. First, while we used Sheridan's 10 levels of automation [11] for decision automation, we did not explicitly specify the number of levels for the other types of automation, e.g., information automation. One reason is that while there is extensive research pointing to the benefits of information automation vs. no automation (e.g., as in predictor displays for CDTI, see [20], [21]), there is as yet little empirical work explicitly comparing the effects on human performance of different levels of automation for information acquisition and analysis. Another reason is that any proposed taxonomy is likely to be superceded by technological developments in methods for information integration and presentation, so that new levels will need to be specified.

Second, in proposing human performance benefits and costs as evaluative criteria for determining appropriate types and levels of automation, we did not discuss how the relative benefits and costs should be weighed. Should the benefit (of a particular automation level) of balanced mental workload be outweighed by the cost of reduced situation awareness or increased likelihood of complacency? What is the relative weighting of the human performance costs we discussed in this paper, as well as of those we did not? Similarly, which is the most important of the several secondary evaluative criteria we have listed, such as automation reliability, costs of action outcomes, ease of system integration, efficiency/safety tradeoffs, manufacturing and operating costs, and liability? These are difficult issues to which there are no simple answers. Of course, as a qualitative model our approach is meant to provide a framework for design, not a set of quantitative methods. Nevertheless, one way forward might be to examine the possibility of formalizing the model. More generally, it would be desirable to have quantitative models that could inform automation design for human-machine systems [77]. Several computational models of human-automation interaction have been put forward very recently, including models based on expected value statistics [37], [78], task-load models [79], cognitive-system models [80], and a model based on state-transition networks [81] (for a recent review of these models, see [82]). As these and related models mature and are validated, it may be possible to improve automation design by supplementing the qualitative analysis presented here with quantitative modeling.

\section{CONCLUSIONS}

Automation design is not an exact science. However, neither does it belong in the realm of the creative arts, with successful design dependent upon the vision and brilliance of individual creative designers. (Although such qualities can certainly help the "look and feel" and marketability of the automated system - see [83]). Rather, automation design can be guided by the four-stage model of human-automation interaction we have proposed, along with the consideration of several evaluative criteria. We do not claim that our model offers comprehensive design principles but a simple guide. The model can be used as a starting point for considering what types and levels of automation should be implemented in a particular system. The model also provides a framework within which important issues relevant to automation design may be profitably explored. Ultimately, successful automation design will depend upon the satisfactory resolution of these and other issues.

\section{ACKNOWLEDGMENT}

The authors thank the members of the Panel on Human Factors in Air Traffic Control Automation of the National Research Council (Anne Mavor, Study Director) for their contributions to this work. They also thank P. Hancock, D. Kaber, N. Moray, U. Metzger, and M. Scerbo for useful comments on this work.

\section{REFERENCES}

[1] E. L. Wiener and R. E. Curry, "Flight-deck automation: Promises and problems," Ergonomics, vol. 23, pp. 995-1011, 1980.

[2] L. Bainbridge, "Ironies of automation," Automatica, vol. 19, pp. 775-779, 1983 
[3] N. Chambers and D. C. Nagel, "Pilots of the future: Human or computer?," Commun. ACM, vol. 28, pp. 1187-1199, 1985.

[4] R. Parasuraman, "Human-computer monitoring," Human Factors, vol. 29, pp. 695-706, 1987.

[5] T. B. Sheridan, Telerobotics, Automation, and Supervisory Control. Cambridge, MA: MIT Press, 1992.

[6] R. Parasuraman and M. Mouloua, Automation and Human Performance: Theory and Applications. Mahwah, NJ: Erlbaum, 1996.

[7] D. D. Woods, "Decomposing automation: Apparent simplicity, real complexity," in Automation and Human Performance: Theory and Applications, R. Parasuraman and M. Mouloua, Eds. Mahwah, NJ: Erlbaum, 1996, pp. 1-16.

[8] R. Parasuraman and V. A. Riley, "Humans and automation: Use, misuse, disuse, abuse," Human Factors, vol. 39, pp. 230-253.

[9] C. E. Billings, Aviation Automation: The Search for a Human-Centered Approach. Mahwah, NJ: Erlbaum, 1997.

[10] C. D. Wickens, A. Mavor, R. Parasuraman, and J. McGee, The Future of Air Traffic Control: Human Operators and Automation. Washington, DC: National Academy Press, 1998.

[11] T. B. Sheridan and W. L. Verplank, "Human and Computer Control of Undersea Teleoperators," MIT Man-Machine Systems Laboratory, Cambridge, MA, Tech. Rep., 1978.

[12] V. Riley, "A general model of mixed-initiative human-machine systems," in Proc. 33rd Annual Human Factors Society Conf.. Santa Monica, CA, 1989, pp. 124-128.

[13] A. D. Baddeley, Working Memory. Oxford, U.K.: Clarendon, 1996.

[14] D. E. Broadbent, Perception and Communication. London, U.K.: Pergamon, 1958.

[15] C. D. Wickens and J. Hollands, Engineering Psychology and Human Performance, 3rd ed. Englewood Cliffs, NJ: Prentice-Hall, 1999.

[16] J. J. Gibson, The Ecological Approach to Visual Perception. Boston, MA: Houghton-Mifflin, 1979.

[17] C. D. Wickens, S. E. Gordon, and Y. Liu, An Introduction to Human Factors Engineering. New York: Longman, 1998.

[18] T. B. Sheridan, "Rumination on automation," in Proc. JFAC-MMS Conf.. Kyoto, Japan, 1998.

[19] J. D. Lee and T. F. Sanquist, Maritime automation in "Automation and Human Performance: Theory and Applications", R. Parasuraman and M. Mouloua, Eds. Mahwah, NJ: Erlbaum, 1996, pp. 365-384.

[20] S. G. Hart and T. E. Wempe, "Cockpit Display of Traffic Information: Airline Pilots Opinions about Content, Symbology, and Format.," NASA Ames Research Center, Moffett Field, CA, NASA Tech. Memo. 78 601, 1979.

[21] M. E. Morphew and C. D. Wickens, "Pilot performance and workload using traffic displays to support Free Flight," in Proc. 42nd Annual Human Factors and Ergonomics Society Conf.. Santa Monica, CA, 1998, pp. 52-56.

[22] N. Moray, "Human factors in process control," in Handbook of Human Actors and Ergonomics, 2nd ed. ed, G. Salvendy, Ed. New York: Wiley, 1997, pp. 1944-1971.

[23] K. Bennett and J. M. Flach, "Graphical displays: Implications for divided attention, focused attention, and problem solving," Human Factors, vol. 34, pp. 513-533, 1992.

[24] A. Mundra, "A New Automation Aid to Air Traffic Controllers for Improving Airport Capacity," The Mitre Corporation, McLean, VA, Technical Report MP-89W00034, 1989.

[25] A. Madni, "The role of human factors in expert systems design and acceptance," Human Factors, vol. 30, pp. 395-414, 1988

[26] E. H. Shortliffe, Computer-Based Medical Consultation: MYCIN. Amsterdam, The Netherlands: Elsevier, 1976

[27] L. L. Schlabach, C. C. Hayes, and D. E. Goldberg, "FOX-GA: A genetic algorithm for generating and analyzing battlefield courses of action," $J$. Evol. Comput., vol. 7, no. Spring, pp. 45-68, 1999.

[28] C. Layton, P. J. Smith, and C. E. McCoy, "Design of a cooperative problem-solving system for en-route flight planning: An empirical evaluation," Human Factors, vol. 36, pp. 94-119, 1994.

[29] W. B. Scott, “Automatic GCAS: You can't fly any lower,” Aviation Week and Space Technology, pp. 76-79, February 1, 1999.

[30] W. Olson and N. B. Sarter, "Supporting informed consent in humanmachine collaboration: The role of conflict type, time pressure, display design, and trust," in Proc. Human Factors and Ergonomics Society 43rd Annual Meeting. Santa Monica, CA, 1999, pp. 189-193.

[31] E. W. Logdson, S. E. Infield, S. Lozito, A. McGann, M. Macintosh, and A. Possolo, "Cockpit data link technology and flight crew communications procedures," in Proc. 8th Int. Symp. Aviation Psychology. Columbus, OH, 1995, pp. 324-239.
[32] E. E. Hahn and J. Hansman, "Experimental Studies on the Effect of Automation on Pilot Situational Awareness in the Datalink ATC Environment," SAE International, PA, Tech. Paper 922 922, 1992.

[33] P. A. Hancock, M. H. Chignell, and A. Lowenthal, "An adaptive humanmachine system," in Proc. 15th Annual IEEE Conf. Syst., Man, Cybern. Washington, DC, 1985, pp. 627-629.

[34] R. Parasuraman et al., "Theory and Design of Adaptive Automation in Aviation Systems," Naval Air Warfare Center, Warminster, PA, Tech. Rep. NAWCADWAR-92 033-60, 1992.

[35] W. B. Rouse, "Adaptive aiding for human/computer control," Human Factors, vol. 30, pp. 431-438, 1988.

[36] M. Barnes and J. Grossman, "The Intelligent Assistant Concept for Electronic Warfare Systems," Naval Warfare Center, China Lake, CA, Tech. Rep. NWC TP 5585, 1985.

[37] T. Inagaki, "Situation-adaptive autonomy: Trading control of authority in human-machine systems," in Automation Technology and Human Performance: Current Research and Trends. Mahwah, NJ: Erlbaum, 1999, pp. 154-158.

[38] E. A. Byrne and R. Parasuraman, "Psychophysiology and adaptive automation," Biol. Psychol., vol. 42, pp. 249-268, 1996.

[39] B. Hilburn, P. G. Jorna, E. A. Byrne, E. A. Byrne, and R. Parasuraman, "The effect of adaptive air traffic control (ATC) decision aiding on controller mental workload," in Human-Automation Interaction: Research and Practice, M. Mouloua and J. Koonce, Eds. Mahwah, NJ: Erlbaum, 1997, pp. 84-91.

[40] D. B. Kaber and J. M. Riley, "Adaptive automation of a dynamic control task based on workload assessment through a secondary monitoring task," in Automation Technology and Human Performance: Current Research and Trends, M. W. Scerbo and M. Mouloua, Eds. Mahwah, NJ: Erlbaum, 1999, pp. 129-133.

[41] N. Moray, T. Inagaki, and M. Itoh, "Adaptive automation, trust, and selfconfidence in fault management of time-critical tasks," J. Exper. Psych. Appl., vol. 6, pp. 44-58.

[42] R. Parasuraman, M. Mouloua, and R. Molloy, "Effects of adaptive task allocation on monitoring of automated systems," Human Factors, vol 38, pp. 665-679, 1996.

[43] S. Scallen, P. A. Hancock, and J. A. Duley, "Pilot performance and preference for short cycles of automation in adaptive function allocation," Appl. Ergon., vol. 26, pp. 397-403, 1995.

[44] M Scerbo, "Theoretical perspectives on adaptive automation," in Automation and Human Performance: Theory and Applications, $\mathrm{R}$. Parasuraman and M. Mouloua, Eds. Mahwah, NJ: Erlbaum, 1996, pp. 37-63.

[45] M. Lewis, "Designing for human-agent interaction," Artif. Intell. Mag., vol. Summer, pp. 67-78, 1998

[46] "Automation surprises," N. Sarter, D. D. Woods, and C. E. Billings, in Handbook of Human Factors and Ergonomics, 2nd ed., G. Salvendy, Ed. New York: Wiley, 1997, pp. 1926-1943.

[47] C. D. Wickens, "Designing for situation awareness and trust in automation," in Proc. IFAC Conf. Integrated Systems Engineering. Baden-Baden, Germany, 1994.

[48] D. D. Woods and N. Sarter, "Evaluating the impact of new technology on human-machine cooperation," in Verification and Validation of Complex Systems, J. A. Wise, V. D. Hopkin, and P. Stager, Eds. Berlin: SpringerVerlag, 1993, pp. 133-158.

[49] K.J. Vicente and J. Rasmussen, "Ecological interface design: Theoretical foundation," IEEE Trans. Syst., Man, Cybern., vol. 22, pp. 489-506, 1992.

[50] E. L. Wiener, "Cockpit automation," in Human Factors in Aviation, E. L. Wiener and D. C. Nagel, Eds. New York: Academic, 1988, pp. 433-461.

[51] A. Kirlik, "Modeling strategic behavior in human-automation interaction: Why "aid" can (and should) go unused," Human Factors, vol. 35 pp. 221-242, 1993

[52] T. K. Landauer, The Trouble with Computers. Cambridge, MA: MIT Press, 1995

[53] D. B. Kaber, E. Omal, and M. R. Endsley, "Level of automation effects on telerobot performance and human operator situation awareness and subjective workload," in Automation Technology and Human Performance: Current Research and Trends. Mahwah, NJ: Erlbaum, 1999, pp. $165-170$

[54] M. Endsley, "Automation and situation awareness," in Automation and Human Performance: Theory and Applications, R. Parasuraman and M. Mouloua, Eds. Mahwah, NJ: Erlbaum, 1996, pp. 163-181.

[55] M. Endsley and E. O. Kiris, "The out-of-the-loop performance problem and level of control in automation," Human Factors, vol. 37, pp. 381-394, 1995 
[56] N. Sarter and D. D. Woods, “'Strong, silent, and out-of-the-loop': Properties of advanced (cockpit) automation and their impact on human-automation interaction," Cognitive Systems Engineering Laboratory, Ohio State University, Columbus, OH, Technical Report CSEL 95-TR-01, 1995.

[57] E. L. Wiener, "Complacency: Is the term useful for air safety?," in Proc. 26th Corporate Aviation Safety Seminar. Denver, CO, 1981.

[58] R. Parasuraman, R. Molloy, and I. L. Singh, "Performance consequences of automation-induced 'complacency'," Int. J. Aviation Psychology, vol. 3, pp. 1-23, 1993.

[59] S. Farrell and S. Lewandowsky, "A connectionist model of complacency and adaptive recovery under automation," J. Exper. Psychol.: Learn., Memory, Cogn., vol. 26, pp. 395-410.

[60] C. D. Wickens, R. Conejo, and K. Gempler, "Unreliable automated attention cueing for air-ground targeting and traffic maneuvering," in Proc. 34th Annual Human Factors and Ergonomics Society Conf.. Santa Monica, CA, 1999.

[61] M. Yeh, C. D. Wickens, and F. J. Seagull, "Target cueing in visual search: The effects of conformality and display location on the allocation of visual attention," Human Factors, vol. 41, 1999.

[62] W. M. Crocoll and B. G. Coury, "Status or recommendation: Selecting the type of information for decision aiding," in Proc. 34th Annu. Human Factors and Ergonomics Society Conf.. Santa Monica, CA, 1990, pp. $1524-1528$.

[63] A. M. Rose, "Acquisition and retention of skills," in Application of Human Performance Models to System Design, G. McMillan, Ed. New York: Plenum, 1989.

[64] A. Swain, "Human reliability analysis: Needs, status, trends, and limitations," Reliab. Eng. Syst. Saf., vol. 29, pp. 301-313, 1990.

[65] D. L. Parnas, A. J. van Schouwen, and S. P. Kwan, "Evaluation of safetycritical software," Commun. ACM, vol. 33, pp. 636-648, 1990.

[66] J. D. Lee and N. Moray, "Trust, control strategies, and allocation of function in human-machine systems," Ergonomics, vol. 35, pp. 1243-1270, 1992.

[67] A. J. Masalonis and R. Parasuraman, "Trust as a construct for evaluation of automated aids: Past and present theory and research," in Proc. Human Factors and Ergonomics Society 43rd Annual Meeting. Santa Monica, 1999, pp. 184-188.

[68] J. A. Swets and R. M. Pickett, Evaluation of Diagnostic Systems: Methods from Signal Detection Theory. New York: Academic, 1982.

[69] J. Kuchar, "Methodology for alerting-system performance evaluation," J. Guidance, Control, and Dyanmics, vol. 19, pp. 438-444, 1996.

[70] R. Parasuraman, P. A. Hancock, and O. Olofinboba, "Alarm effectiveness in driver-centered collision-warning systems," Ergonomics, vol. 39, pp. 390-399, 1997.

[71] J. L. Merlo, C. D. Wickens, and M. Yeh, "Effects of reliability on cue effectiveness and display signalling," University of Illinois Aviation Research Lab, Savoy, IL, Technical report ARL-99-4/Fedlab-99-3, 1999.

[72] “Aviation Week and Space Technology," Answers to the Gridlock, pp. 42-62, February 2, 1998

[73] "Report of the RTCA Board of Director's Select Committee on Free Flight," RTCA, Washington, DC, 1995.

[74] R. van Gent, J. M. Hoekstra, and R. C. J. Ruigrok, "Free flight with airborne separation assurance," in Proc. Int. Conf. Human Computer Interaction in Aeronautics. Montreal, , Canada, 1998, pp. 63-69.

[75] P. M. Fitts, Human Engineering for an Effective Air Navigation and Traffic Control System. Washington, DC: National Research Council, 1951.

[76] T. B. Sheridan, "Allocating functions rationally between humans and machines," Ergonomics in Design, vol. 6, no. 3, pp. 20-25, 1998.

[77] R. W. Pew and A. S. Mavor, Modeling Human and Organizational Behavior: Application to Military Simulations. Washington, DC: National Academy Press, 1998

[78] T. B. Sheridan and R. Parasuraman, "Human vs. automation in responding to failures: An expected-value analysis," Human Factors, to be published.

[79] Z. Wei, A. P. Macwan, and P. A. Wieringa, "A quantitative measure for degree of automation and its relation to system performance," Human Factors, vol. 40, pp. 277-295, 1998.

[80] K. Corker, G. Pisanich, and M. Bunzo, "Empirical and analytic studies of human/automation dynamics in airspace management for free flight," in Proc. 10th Int. CEAS Conf. Free Flight. Amsterdam, The Netherlands, 1997.
[81] A. Degani, M. Shafto, and A. Kirlik, "Models in human-machine systems: Constructs, representation, and classification," Int. J. Aviation Psychology, vol. 9, pp. 125-138, 1999.

[82] R. Parasuraman, "Designing automation for human use: Empirical studies and quantitative models," Ergonomics, to be published.

[83] D. A. Norman, The Invisible Computer, 1998.

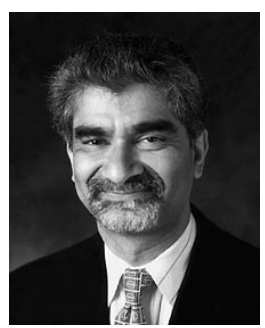

Raja Parasuraman received the B.Sc. degree (first class honors) in electrical engineering from Imperial College, University of London, U.K. in 1972, and the M.Sc. and Ph.D. degrees in applied psychology from the University of Aston, Birmingham, U.K in 1973 and 1976, respectively.

From 1978 to 1982, he was a Research Fellow at the University of California, Los Angeles. In 1982, he joined the Catholic University of America, Washington, D.C. as Associate Professor and was promoted to Full Professor in 1986. He is currently Director of the Cognitive Science Laboratory and also holds a visiting appointment at the Laboratory of Brain and Cognition at the National Institute of Mental Health, Bethesda, MD. His research interests are in the areas of attention, automation, aviation and air traffic control, event-related brain potentials, functional brain imaging, signal detection, vigilance, and workload.

Dr. Parasuraman is a Fellow of the American Association for the Advancement of Science (1994), the Human Factors and Ergonomics Society (1994), and the American Psychological Society (1991). He is also currently serving on the National Research Council Panel on Human Factors.

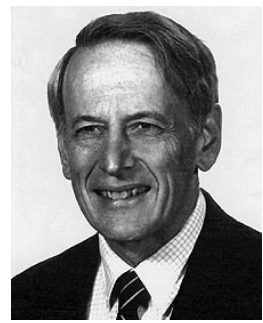

Thomas B. Sheridan (M'60-SM'82-F'83-LF'96) received the B.S. degree from Purdue University, West Lafayette, IN, the MS degree from University of California, Los Angeles, the Sc.D. degree from the Massachusetts Institute of Technology (MIT), Cambridge, and the Dr. (honorary) from Delft University of Technology, The Netherlands.

For most of his professional career he has remained at MIT, where he is currently Ford Professor of Engineering and Applied Psychology Emeritus in the Department of Mechanical Engineering and Department of Aeronautics and Astronautics, continuing to teach and serve as Director of the Human-Machine Systems Laboratory. He has also served as a visiting professor at University of California, Berkeley, Stanford, Delft University , Kassel University, Germany, and Ben Gurion University, Israel. His research interests are in experimentation, modeling, and design of human-machine systems in air, highway and rail transportation, space and undersea robotics, process control, arms control, telemedicine, and virtual reality. He has published over 200 technical papers in these areas. He is co-author of Man-Machine Systems (Cambridge, MA: MIT Press, 1974, 1981; USSR, 1981), coeditor of Monitoring Behavior and Supervisory Control (New York: Plenum, 1976), author of Telerobotics, Automation, and Human Supervisory Control (Cambridge, MA: MIT Press, 1992), and co-editor of Perspectives on the Human Controller (Mahwah, NJ: Erlbaum, 1997). He is currently Senior Editor of the MIT Press journal Presence: Teleoperators and Virtual Environments and serves on several editorial boards. He chaired the National Research Council's Committee on Human Factors, and has served on numerous government and industrial advisory committees. He is principal of Thomas B. Sheridan and Associates, a consulting firm.

Dr. Sheridan was President of the IEEE Systems, Man, and Cybernetics Society, Editor of IEEE TRANSACTIONS ON MAN-MACHINE SYSTEMS, received their Norbert Wiener and Joseph Wohl awards, the IEEE Centennial Medal and Third Millenium Medal. He is also a Fellow of the Human Factors and Ergonomics Society, recipient of their Paul M. Fitts Award, and was President of HFES. He received the 1997 National Engineering Award of the Amer.ican Association of Engineering Societies and the 1997 Oldenburger Medal of ASME. $\mathrm{He}$ is a member of the National Academy of Engineering. 


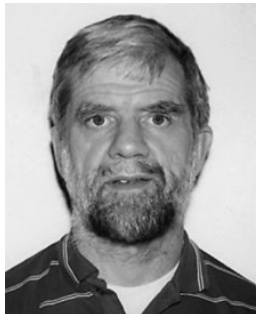

Christopher D. Wickens received the A.B. degree from Harvard College, Cambridge, MA, in 1967 and the Ph.D. degree from the University of Michigan, Ann Arbor, in 1974.

He served as a commissioned officer in the U.S. Navy from 1969 to 1972 . He is currently a Professor of Experimental Psychology, Head of the Aviation Research Laboratory, and Associate Director of the Institute of Aviation at the University of Illinois at Urbana-Champaign. He also holds an appointment in the Department of Mechanical and Industrial Engineering and the Beckman Institute of Science and Technology. His research interests involve the application of the principles of human attention, perception and cognition to modeling operator performance in complex environments, particularly aviation, air traffic control and data visualizations.

Dr. Wickens is a member and Fellow of the Human Factors Society and received the Society's Jerome H. Ely Award in 1981 for the best article in the Human Factors Journal, and the Paul M. Fitts Award in 1985 for outstanding contributions to the education and training of human factors specialists. He was elected to the Society of Experimental Psychologists, elected Fellow of the American Psychological Association, and in 1993 received the Franklin Taylor Award for Outstanding Contributions to Engineering Psychology from Division 21 of that association. 